\title{
DESIGN AND IMPLEMENTATION OF ONLINE EXAMINATION ADMINISTRATION SYSTEM FOR UNIVERSITIES
}

PRINCE ANA AND PAUL TAWO BUKIE

\begin{abstract}
Online examination system is a web-based examination system where examination is taken online i.e. through the internet or intranet using computer system. It is an effective solution for mass education evaluation. We have developed an online examination system based on a Browser/Server framework using Microsoft Visual Studio 2008 for the design, C\# 3.5 for coding, and Microsoft SQL Server as database. ASP.NET is the preferred web technology. The system carries out the examination and auto-grading for multiple choice questions which is feed into the system.
\end{abstract}

KEY WORDS: Online, Examination, System, Auto-grading, Web-based.

\section{INTRODUCTION}

Examination, they say is not a true test of knowledge. In our contemporary society, this axiom holds only in theory but not in practice. Examination that is supposed to be conducted and evaluated in confidence is now seen with students even before the date and time the examination is scheduled to take place.

History has it that ancient China; was the first country in the world that implemented a nationwide standardized examination, which was called the "imperial examination". The main purpose of this examination was to select able candidates for specific governmental positions. The imperial examination was established by the Sui Dynasty in 605 AD and was later abolished by the Qing Dynasty 1300 years later at 1905 . England adopted this examination system in 1806 to select specific candidates for positions in Her Majesty's Civil Service. This examination system was later applied to education and it started to influence other parts of the world as it became a prominent standard (e.g. regulations to prevent the markers from knowing the identity of candidates), of delivering standardized tests.

There are three methods of examination: written examinations, oral examinations and physical fitness examination. In written examinations we have the multiple choice questions. Multiple choice questions have two sub categories. The first category is called True/False. This requires the student to choose all answers that are appropriate. True/False questions present candidates with a binary choice - a statement is either true or false. This method presents problems, as depending on the number of questions, a significant number of candidates could get one hundred percent (100\%) just by guesswork, and should on average get fifty percent (50\%). The second category is called BestAnswer question. This requires the student to only answer from a list of options. Other forms of questions in written examinations include:
Matching - a matching item is an item that provides a defined term and requires a test taker is to match identifying characteristic to the correct term.

Fill-in-the-Blank - a fill-in-the-blank item provides a student with identifying characteristics and requires the student to recall the correct term.

Essay - in essay, an item is given to a student. Essay typically requires a student to write a response to fulfill the requirements of the item. In administrative terms, essay items take less time to construct. As an assessment tool, essay items can test complex learning objectives as well as processes used to answer the question. The items can also provide a more realistic and generalized task for examination.

Mathematical questions - most mathematics questions, or calculation questions from subjects such as chemistry, physics or economics employ a style which does not fall in to any of the above categories. Instead, most mathematics questions simply state a question and require the candidate to solve it, usually with marks given more for the steps taken than for the correct answer.

Oral Examination - is a type of examination where the student is tested based on his ability to face a panel and answer questions by speaking. Assessment is based on how much the student can comport his/herself.

Physical Fitness Examination - is a test designed to measure physical strength, agility, and endurance. They are commonly employed in educational institutions as part of the physical education curriculum, in medicine as part of diagnostic testing, and as eligibility requirements in fields that focus on physical ability such as military or police. 


\section{BACKGROUND OF THE STUDY}

Manual Examination System is replete with several problems. A cursory glance at Manual Examination Administration System reveals cases of examination malpractice and other immoral acts committed by students, which violates the rules and regulations of Universities as it concerns the administration of examination as well as the human moral content. This has taken a tool from the Nation, as it produces half-baked graduates who cannot compete with University graduates from other nations of the world in the labor market. The need to protect the image of the Nigerian nation as well as the University System is very pertinent.

In furtherance, during manual computation of results, errors may arise due to "omission" or "commission". There are also cases of missing examination scripts during marking. There is also a problem of time wasting, among others.

This research suggests that if an efficient and effective online examination system is developed, where examinations will be taken online, and results computed and released immediately, as well as stored in a central database for documentation and future planning and evaluation purposes, there shall be relative balance and harmony within the University System. The staff will take the advantage of time that would have been spent on marking examination and preparing results to enable them do their research work. This will also cause the unserious students to be committed to their studies.

\section{MATERIALS AND METHODS}

With respect to system analysis and design, methodology refers to the documentation of approaches which are used to handle activities in a coherent, consistent, accountable and repeatable manner. Methodology is a process that mainly consists of intellectual activities usually only the end goal of the methodology process is manifested as the product or result of the physical work.

In software, the term methodology is used to refer to series of steps or a procedure which governs the activities of analysis and guidelines to design or an organized documented set of procedures and guidelines for one or more phases of the (software life cycle), such as analysis or design.

The methodology adopted for this research is Structured Systems Analysis and Design Methodology (SSADM). We shall also give cogent reason for adopting this methodology.

SSADM is a very popular methodology used in the analysis and design stages of systems development. It adopts a prescriptive approach to information systems development in that it specifies in advance the modules, stages and task which have to be carried out, the deliverables to be produced and furthermore, the techniques used to produce the deliverables.

SSADM adopts the "Waterfall Model" of system development life cycle, where each phase has to be completed and signed off before subsequent phases can begin. This model is named "Water Fall" because its diagrammatic representation resembles a cascade of waterfalls (the natural wonders of falling water). This model is easy to understand and reinforces the notion of "define" and "design" before "code".
The water fall model is one of the earliest attempts to describe the software development life cycle. In the waterfall model, the project is split up into phases: requirements capture, analysis, design, implementation, testing, maintenance, etc., i.e. development moves from concept, through analysis, design, implementation, testing, installation, troubleshooting, and ends up at operation and maintenance. Each phase of development proceeds in strict order, without any overlapping or iterative steps. In the strict case, each phase must be finished before development proceeds to the next phase. SSADM revolves around the use of three key techniques, namely: Logical Data modeling, Data Flow Modeling and Entity Event Modeling.

\section{Logical Data Modeling (LDS)}

This is the process of identifying, modeling and documenting the data requirements of an information system. A logical data flow model consists of a logical data structure and the associated documentation. LDS represents Entities and Relationships.

\section{Data Flow Modeling (DFM)}

This is the process of identifying, modeling and documenting how data flows around an information system. A data flow model consists of a set of integrated data flow diagrams supported by appropriate documentation. DFMs represent processes (activities which transforms data from one form to another), data stores (holding areas for data), and external entities (things which send data into a system or receive data from a system) and finally data flows (routes by which data can flow through).

\section{Entity Event Modeling (EEM)}

This is the process of identifying, modeling and documenting the events which affects each entity and the sequence in which these events occur. An EEM consists of a set of entity life histories (one for each entity and appropriate supporting documentation.

With respect to the above, SSADM provides a flow or a water fall view of systems development, which has steps. Each step leads to the next step. The SSADM steps or stages are: Feasibility Study, Requirement analysis, Requirements specifications, Logical system specifications and Physical design.

The materials used in this research work are as follows:

\section{Visual Studio 2008}

Visual Studio 2008 is a suite that contains the Microsoft .NET framework and a variety of other programming languages that programmers can choose from such as. This position is supported by William B Sanders in his book entitled "ASP.NET 3.5: A Beginner's Guide" when he posited thus:

"For those using ASP.NET for the first time, the good news is that the transition to the server side could hardly be easier. Everything in this book is set up to learn ASP.NET 3.5 and C\# 3.0 using Visual Studio 2008. Using the tools built into Visual Studio 2008, you will quickly 
learn that most of what needs to be done can be accomplished by dragging controls into a visual editor. And with the "code behind" method, C\# is added in a separate file, so when editing code, you will see a clear separation-the C\# 3.0 code comes "behind" the ASP.NET 3.5 code".

Jesse Liberty and Hurwitz in their book entitled "Programming ASP.NET" edited by Ron Petrusha and Valerie Quercia opined that "ASP.NET is the name Microsoft has given to the combination of its two Web Development technologies: Web Forms and Web Services. Using ASP.NET, it is easier than ever to create web applications that are dynamic and datadriven, that scale sell, and that work well across a broad range of browsers without any custom coding by the developer".

To support William B Slander in his opinion of the variety of programming languages in the Visual Studio 2008, they went further to uphold that "the .NET Framework consists of a number of components, prominent among which are:

Four official languages: C\#, Visual Basic NET, Managed $\mathrm{C}++_{+}$, and Jscript.NET. It supports a common language specification (CLS) that allows you to choose the syntax with which you are most comfortable. The choice of language is a personal preference rather than a limiting factor in your applications as well.

The Common Language Runtime (CLR), an object oriented platform for windows and web development that all these languages share. The CLR executes your program on your web server. The CLR activates objects, performs security checks on them, lays them out in memory, executes them, and handles garbage collection.

A number of related class libraries, collectively known as the framework class libraries. The set of framework base class support rudimentary input and output, string manipulation, security management, network communication, thread management, text manipulation, reflection and collections functionality, etc. (Jesse Liberty and Hurwitz, 2002)

Stephen Walther (2008) in his work entitled "ASP.NET 3.5 Unleashed" applaud ASP.NET 3.5 when he writes "ASP.NET is Microsoft's flagship technology for building highly interactive, highly scalable websites. Some of the largest websites hosted on the Internet were built with the ASP.NET Framework, including Dell (www.Dell.com), MySpace (www.MySpace.com), and Microsoft (www.Microsoft.com). If you need to build a highly interactive website that can scale to handle thousands of simultaneous users, then ASP.NET is the technology to use".

Visual Studio 2008 has a toolbox which contains in-build pre-defined functions, objects like text boxes, labels, HTML, pointers, dropdown list, and link buttons, radio buttons and a lot of other controls that programmers can just drag and drop and then manipulate the codes. It supports code refactoring. It also have a property bar where programmers can easily assign properties to a particular object. The application interface have the design side - which handles the Graphic User Interface and the source side - which handles the program code. While working, you can split the application interface to enable you view both the design and source sides in one application window.

\section{ASP.NET and the .NET Framework}

ASP.NET is part of the Microsoft .NET Framework. To build ASP.NET pages, you need to take advantage of the features of the .NET Framework. The .NET Framework consists of two parts: the Framework Class Library and the Common Language Runtime, Stephen Walther (2008).

ASP.NET introduces runtime services and a well-engineered class library for greatly enhancing Web development. In a way, classic ASP was a sort of "taped onto" the IIS/ISAPI architecture without any real organic thought as to how early design decisions would affect developers later on.

ASP.NET is built from the ground up to be an extensible, feature-rich way to handle HTTP requests. ASP.NET leverages IIS in that requests for ASP.NET services are mapped to an ISAPI DLL. The DLL is named ASPNET_ISAPI.DLL. From there, processing is passed into a worker process provided by ASP.NET (ASPNET_WP.EXE in IIS 5 or W3WP.EXE in IIS 6). The fundamental request processing is handled by managed types within the worker process. Control passes between a number of classes plugged into the pipelinesome provided by Microsoft and/or third parties, and some provided by the developer.

ASP.NET is built from the ground up to be a comprehensive framework for writing Web applications. All the parts of the framework execute together to handle requests. By contrast, classic ASP.NET script code had no structure to it, and code logic within applications tended to be ad hoc (George Shepherd, 2005)

ASP.NET provides a significant number of very powerful features, including: An object-oriented framework for defining applications, Separation of user interface declarations (HTML) and application logic, Compiled code for executing application logic, Configurable session state management, Built-in data caching, Builtin content caching, A well-defined UI componentization architecture, High-level components for managing data formatting (grids, lists, text boxes), Built-in program tracing and diagnostics, Built-in user input validation, An easy-to-use custom authentication mechanism, Solid integration with ADO.NET (the .NET database story), Excellent support for Web Services, Zero reliance on the Component Object Model, An extensible pipeline with many places in which a request can be intercepted (George Shepherd, 2005)

Other features in recent versions of ASP.NET are as follows: Master Pages and Skins, Declarative databinding, Provider pattern model, New cache features, Membership controls, Personalization controls, Support for Web Parts, Programmable configuration, Administration tools, New compilation model. However, these new features make ASP.NET an even more compelling platform for creating Web sites... (George Shepherd, 2005)

$\mathrm{C \#}$ is a language targeted at developers for the Microsoft .NET platform who have already worked with a 
C-like language such as $\mathrm{C}, \mathrm{C}++$, or Java. Unlike previous versions of $\mathrm{C}$ or $\mathrm{C}++$ for the Microsoft Windows platform, C\# code runs under a managed execution environment. Microsoft portrays $\mathrm{C \#}$ as a modern and innovative language for .NET development and continues to deliver on that with new features such as Language Integrated Query (LINQ). The new features in C\# 3.0 allow for more of a declarative and functional style of programming, when that is appropriate, while it still has great object-oriented features as well. The main idea is to use the style of programming that fits your problem, and C\# will support your endeavor (Jay Hilyard and Stephen Teilhet, 2008).

C\# allows you to perform many $\mathrm{C} / \mathrm{C}++-$ like functions, such as direct memory access via pointers and operator overloading, that are not supported in Visual Basic .NET.

$\mathrm{C \#}$ is the system-level programming language for .NET. You can still do great application-level work in C\#, (Jay Hilyard and Stephen Teilhet, 2008).

If you have seen C\#, you may have noticed that it looks a lot like Java; Java programmers will feel very much at home in C\# once they learn the Framework SDK. C\# can also be a great language for Visual Basic .NET programmers when they need a little more control over what the code is doing and don't want to have to write $\mathrm{C}++$ to gain an advantage.

\section{SQL Server 2005}

SQL Server 2005 brings with it a vast array of new features, graphical user interfaces (GUIs), and management tools. The following list should give you a brief taste of these:

- The ability to host the .NET Framework common language runtime (CLR) in the database so that you can now program assemblies in Visual Basic 2005 and C\# in the database. This may have interesting consequences for the SQL Server database programmer, who previously was limited to SQL and T-SQL, and it will have dramatic implications for the way applications may be architected.

- Deep support for XML, via a full-fledged XML data-type that carries all the capabilities of relational data types. You can enter an XML document into your database, have it validated, and extract just part of the document. This means that you can marry semi-structured data with relational data, storing them in the same place and treating them in the same way. Additionally, server-side support is provided for XML Query (XQuery) and XML Schema Definition language (XSD) standards.

- A completely revamped GUI management tool called SQL Server Management Studio (SSMS), which provides a single, integrated environment for most management/administration requirements.

- A reporting framework (SQL Server Reporting Services, or SSRS) as an integral part of the database.
- A new application framework, the Service Broker, for asynchronous message delivery.

- Vastly improved and expanded SQL Server Integration Services (SSIS; formerly Data Transformation Services), a tool for extracting, transforming, and loading data (again, a feature that is a costly add-on with other relational database management systems).

The latter three are excellent examples of features that SQL Server provides as an integral part of the product, rather than as (extra-cost) add-ons.

Microsoft SQL Server 2005 comes in a variety of editions and each of these editions brings with it, advancement over the previous one. Simply put, editions are designed to handle specific tasks. (Thomas Rizzo, Adam Machanic, Julian Skinner, Louis Davidson, Robin Dewson, Jan Narkiewicz, Joseph Sack, Rob Walters (2006).

However, Microsoft SQL Server 2005 is available in the following distinct editions:

- Enterprise: This is the most powerful, scalable, and expensive SQL Server 2005 edition. It is targeted, as its name suggests, at enterprise businesses where performance availability and scalability are of paramount importance. It supports all available features.

- Developer: This is the same as the Enterprise Edition, but with restrictions on CPUs and licenses.

- Standard: This edition is a cheaper option than Enterprise and Developer, and it is targeted at smalland medium-sized businesses. It removes support for such features as partitioning and online indexing, but it does support many of the "high-end" features, such as Analysis Services, Integration Services, database mirroring, and so on.

- Workgroup: This edition is designed for small- and medium-sized businesses and departmental solutions. It supports many of the core SQL Server features, but it doesn't include high-availability features, and it also has limited analysis functionality.

- Express: This edition replaces Microsoft SQL Server Desktop Engine (MSDE). However, it inherits many (non-enterprise) features from SQL Server 2005 and comes complete with its own dedicated (albeit limited) development and administration tools. It is freely available and is an ideal database for departmental solutions, prototype or evaluation projects, and hobbyists (Thomas Rizzo, Adam Machanic, Julian Skinner, Louis Davidson, Robin Dewson, Jan Narkiewicz, Joseph Sack, Rob Walters (2006).

\section{Features of the Application Randomised Exams}

The system is designed to randomise questions for candidates taking the exams. For instance, if twenty candidates are taking the exams, candidate number one may be served with question number 4 as question 1 while candidate number 40 will be furnished with question number 20 as question 1 . So, no two 
candidates can have the same question number furnished as question 1.

\section{Timer}

The application has a timer to regulate the examination time for each candidate taking the examination.

\section{The Preparation System}

The preparation system is used to manage question storage, assign test ID and schedule the test. The question database is composed of the questions, a set of possible answers, the question types and other metadata, which are indexed by several factors, such as topics, keywords, complexity and difficulty, etc. The database is open to administrator, allowing him/her to add questions and answers.

\section{The Examination System Can Be Both Web-Based And On An Intranet Or An Adhoc Network}

The examination System is a web-based testing interface for students. It include the following key features: client side control, time control, security control and auto-installation. Because the application benefits from COM technology of Microsoft, the system can install and update itself conveniently. Becsause the system is established on the Web, the systematic security faces a severe challenge. We guarantee the security by cryptography, real-time monitoring system and data transmission encryption. The cryptography is used to validate student identification before testing.

\section{The Real-Time Monitoring System}

The real-time monitoring system requires students not to leave the computer during the test by face tracking technology. The data transmission encryption system transmits the examination question and result in secret form through the network to the server. The examination monitor system is also the manager of the examination system, by which we can monitor the test processing, carry out test ID statistic and collect the answers, etc.

\section{The Auto-Grading System}

The auto-grading system is designed by the fuzzy matching algorithm and the macro programming technology, which is seasoned with variable question types.

\section{DISCUSSION ON THE ANALYSIS OF FUNCTIONALITY OF THE SYSTEM}

The system is developed using Microsoft Visual Studio 2008. Microsoft Visual Studio 2008 works with a technology called ASP.NET. The actual coding was done using $\mathrm{C \#}$. The system have a three tier architecture which are the front-end (user interface), back-end (the administration) and the database (core store). The front end was developed using $\mathrm{C \#}$, the core store was developed using Microsoft SQL Server 2005, .Net framework was used as the

linking technology as well as Cascading Style Sheet (CSS) and HTML. The entire system is designed to be web based, such that it can be launched into the internet.

The system has seven modules. These modules are split up into two categories: Administrator and Student. The Administrator has five modules while the student has two modules. However, the timer and logout modules appear on both the Administrator and student sections.

Just like in every other conventional method of administering examination, for a student to qualify to take this exam, such a student must register for exams in the Administrator's office. During registration, the Administrator generates username, password and a PIN for each student. This PIN is like the student's Identification (ID). The student is also given the examination time and date. On the examination day, the student login to the website and then enters his/her PIN to enable him/her take the exams.

However, if a student enters a wrong PIN, the system prompts him/her with a message "The PIN you entered does not exist". Furthermore, if a student enters string values in the Enter Exam PIN space on the system, the system will prompt him/her with a message "Numeric values only needed." If the student clicks write exams without entering the PIN, the system prompts the student with a message "Please Enter PIN".

Alternatively, depending on the decision of the University management, the can as well generate PIN and pack the PIN in the form of scratch cards. These PINs are sold to students in designated points, banks, more preferably. The students login to the website and then enter the PIN in the Enter Exam PIN space on the system, to enable them write the exams using. But, this will require the to change the configuration of the system functionality as regards security and authentication.

One thing worthy of note here is that, ones the student write the exams and click submit, the PIN assigned to him will automatically be destroyed and rendered useless.

\section{An Appraisal of the System Design}

System design is the process or act of defining the hardware or software architecture, components, modules, interface and data for a computer system to satisfy specified requirements. One could see it as the application of system theory to computing. Some overlap with the discipline of system analysis appears inevitable. Nothing can guarantee that an automated system selection process will be successful, but adherence to a set of commonsense principles can help in securing a successful outcome. The focus of the process has been on a long term and has taken into account, the institutional context into which the system will fit. With the shift towards user empowerment, the involvement of users in the selection process is increasingly critical.

The components of the selection process can be envisioned and combined in many different ways. This is shown in the way the codes used were broken into four major modules and later reassembled together by creating a linking technology. Each of these modules contains particular ASP.NET base class libraries. The modules are discussed below: 
Logout

Logout enables the student to return to the login page, after writing the exams. The admin can also logout after performing any task on the system.

\section{Create Exams}

Create exams as the name implies enable admin to create exams. This module is strictly for the admin. The admin clicks on the link, enter exams name, enter the score allocated to the exams as well as the pass score for the exams and then clicks submit

\section{Create Questions}

Create questions as the name implies enable admin to create questions. The user clicks on the link, select the exams he/she wants to create questions for, type the question number, input the question, input the options and then input the correct option and finally clicks submit.

\section{Register Student}

For any user/student to be eligible for the examination, such a user/student must be registered. During registration, a student fill a form and upload his/her photograph. The user/student is also given login credentials i.e. a username, password and a PIN. These login credentials are used by the student to write exams.

We have used the Random Class to implement the "Generate PIN". The function of the random class is to generate the PIN. I created an instance of the random name. The reason for this is to enable me get access to members of the class. By members of the class, I mean the properties of the class. The instance name is $\mathrm{rdn}$. The property name is Next. Hence, rdn.Next.

Random rdn = new Random();

Int PIN = rdn.Next $(0000,9999)$;

\section{View Result}

Here, a score sheet that comprise results of all the users/students that took the exams can be viewed in one document. It can as well be printed or saved.

\section{Timer}

The timer was implemented using AJAX (Asynchronous JavaScript and XML). There is an AJAX tool in Visual Studio 2008 called Timer. For the timer to work, I embedded what we call Script Manager. As the name implies, Script Manager manages all AJAX functionality in any web page that contains an AJAX tool.

The main function of the timer in this application is to time as well as guide the students during exams to be time conscious.

The timer enables users to see the time allocated to the entire examination. The timer is programmed to start counting from 1000 and decrement to 0 . When the timer decrements to 0 , the system log user out. One thing worthy of note here is that, such users will not have any record in the database i.e. their preliminary result will not be displayed. The PIN is also destroyed.

\section{Write Exams}

To write the exams, the student clicks on the user link on the top menu. The student login with the username and password assigned during registration. The student is further prompted to enter the PIN generated by admin during registration. After logging in, the student chooses the exam type and proceeds to answer the questions presented one after the other by clicking next button. When the student finish answering the questions, the student clicks on the submit button. On clicking the submit button, the student is presented with the preliminary result immediately.

\section{An Appraisal of the Database Design}

The database was designed with Microsoft SQL Server 2005. The database itself consists of four simply designed tables. As you view the table, you will notice that the primary key for the database, a compound key, was selected as unique identifier. The entities and relationships in the main codes maps directly to object classes and named relationship.

The first table contains information about the student. It contains seven fields. They are UserName, PassWord, ExamPin, MatricNo, Name, Phone, Email, ImageUrl, and RegisteredDate.

The second table has information about Exams. It has four fields. They include: Examld, ExamName, ExamScore, PassingScore.

The third table keeps information about Score. It has seven fields. These fields include the following: Scoreld, ExamName, MatricNo, TestDate, PassingScore, StudentScore and StudentName.

The forth and last table houses information about the questions. It has ten fields. The fields include: Questionld, Examld, QuestionNo, Question, OptionA, OptionB, OptionC, OptionD, CorrectAnswer as well as Mark. 


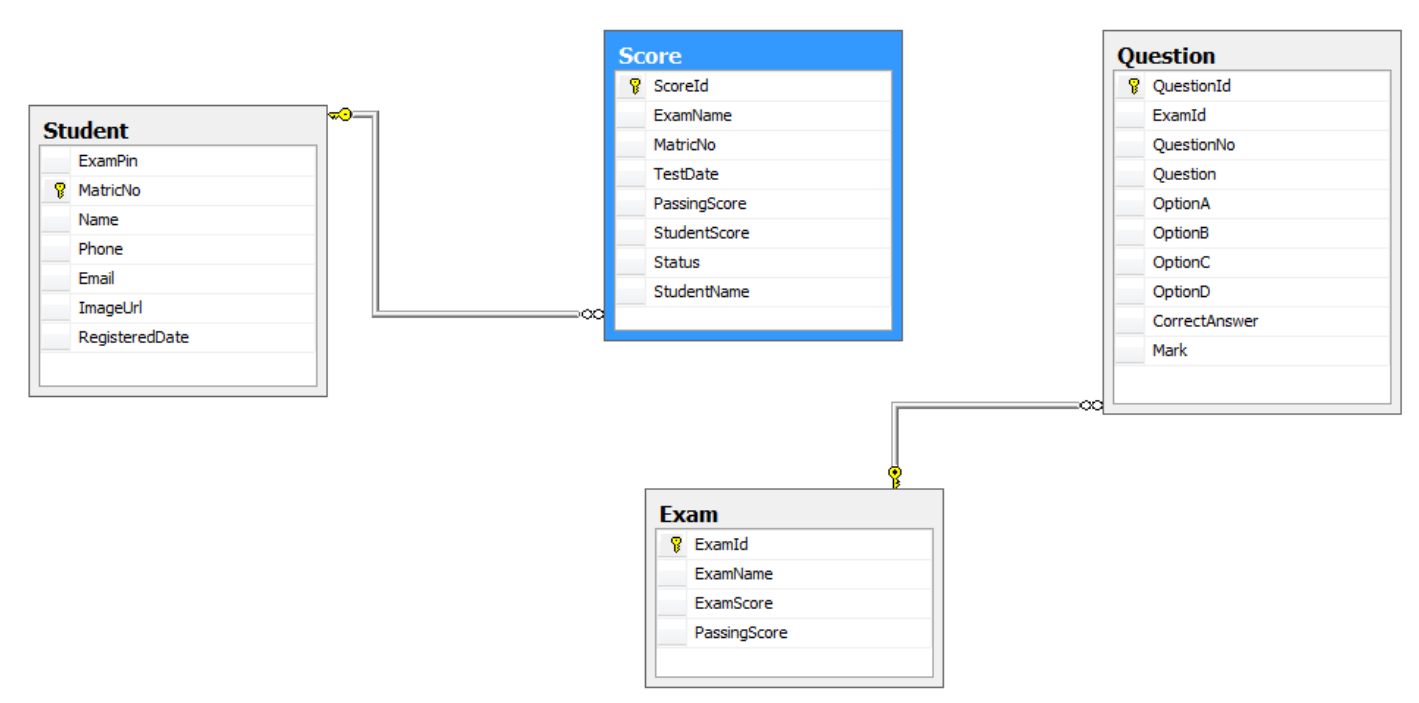

Fig.1.3. Diagram of the Database Design

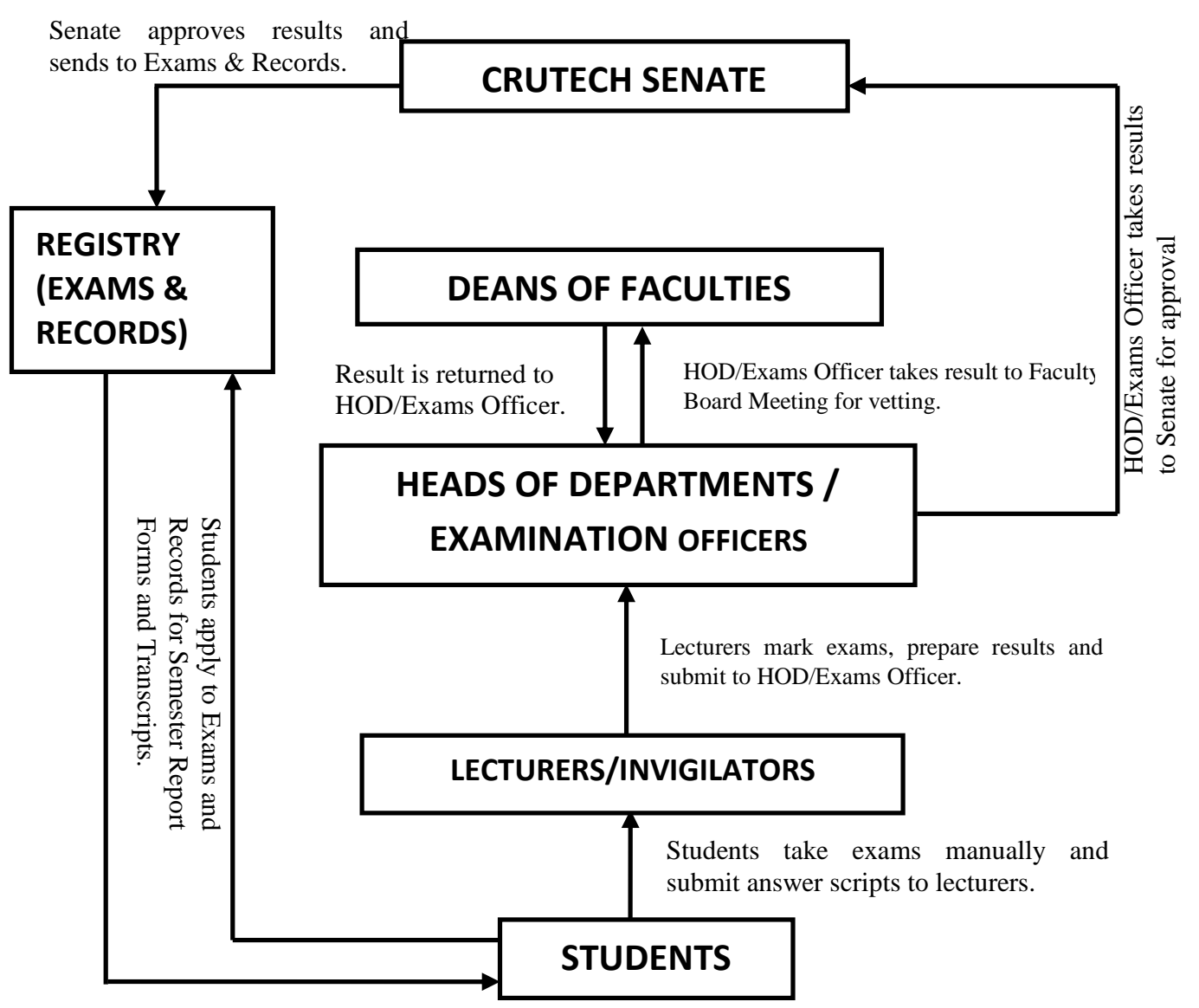

Fig.1.1: Functional Diagram of the Existing System 


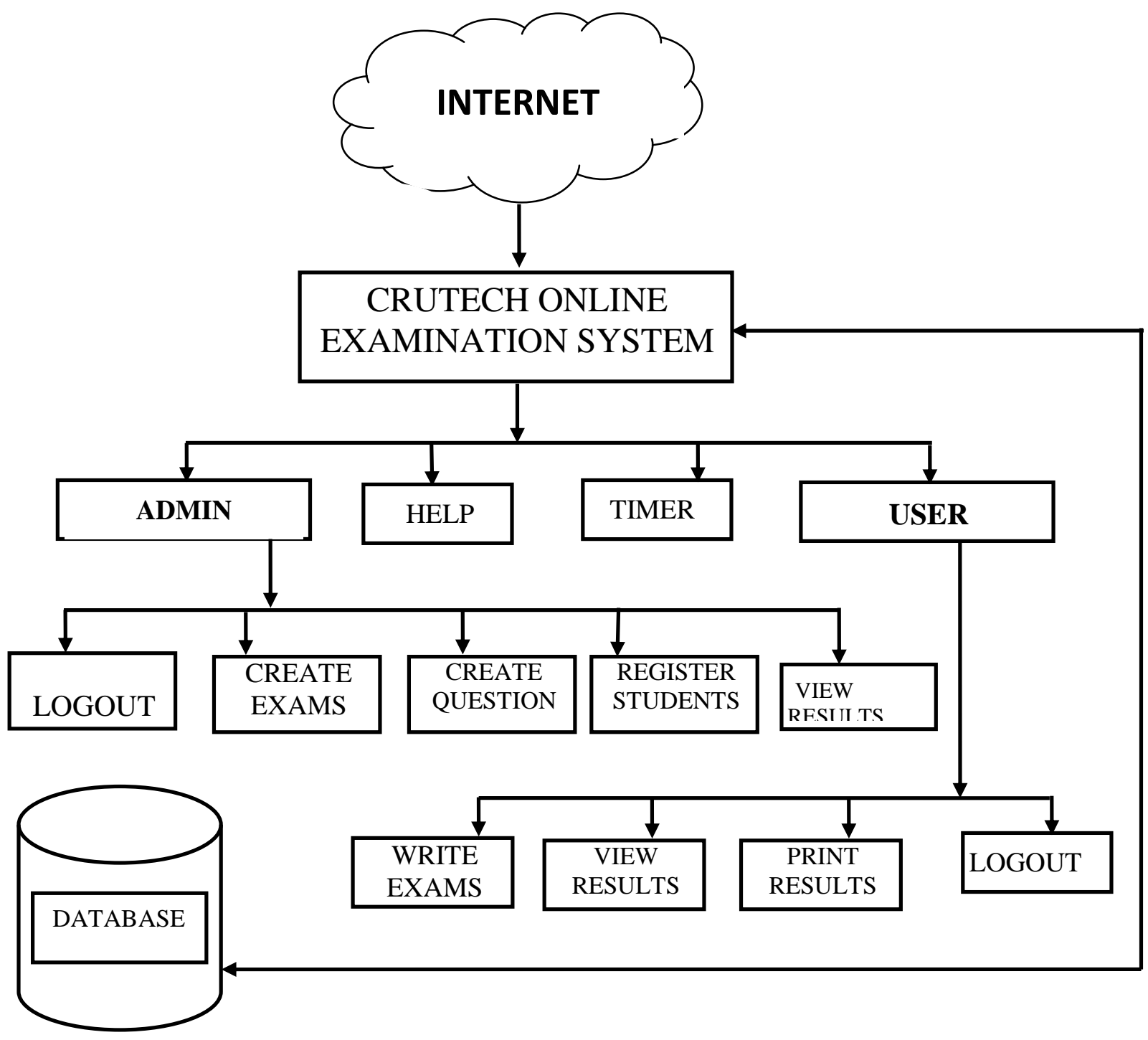

Fig.1.2: High Level Model Diagram for the Proposed System 


\section{SAMPLE OUTPUT OF PROPOSED SYSTEM}

\section{Home Pages}

This is the first page you see when you login to the application. It has a welcome message and Admin and User links on the top menu.

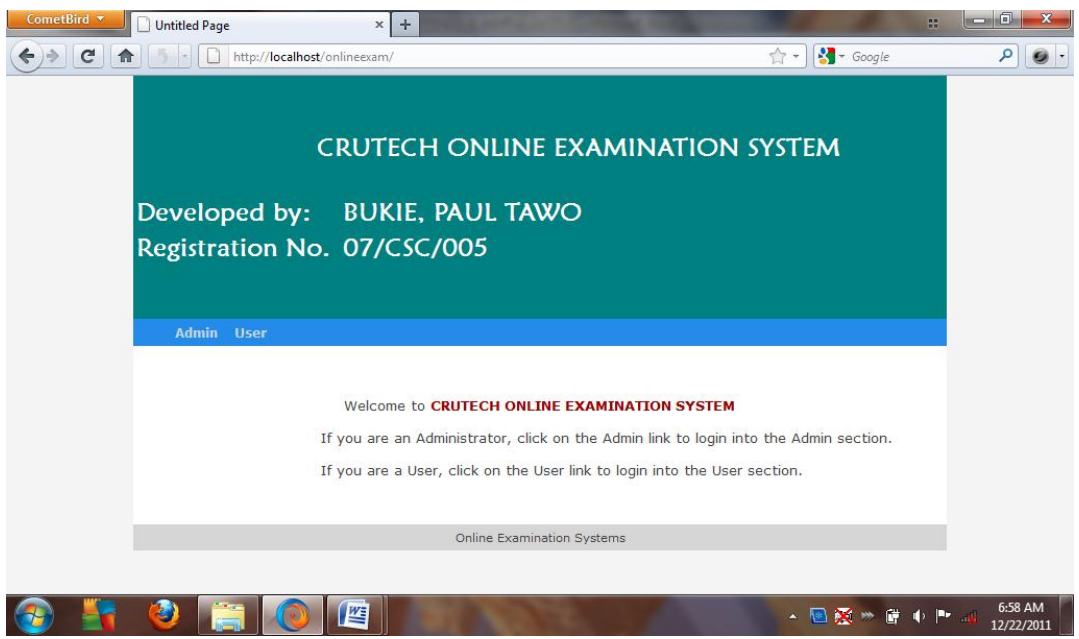

\section{Administrator Login Screenshot}

This page allows administrator to enter his login credentials to enable him/her create exams, create questions, register students, view scores and then log out.

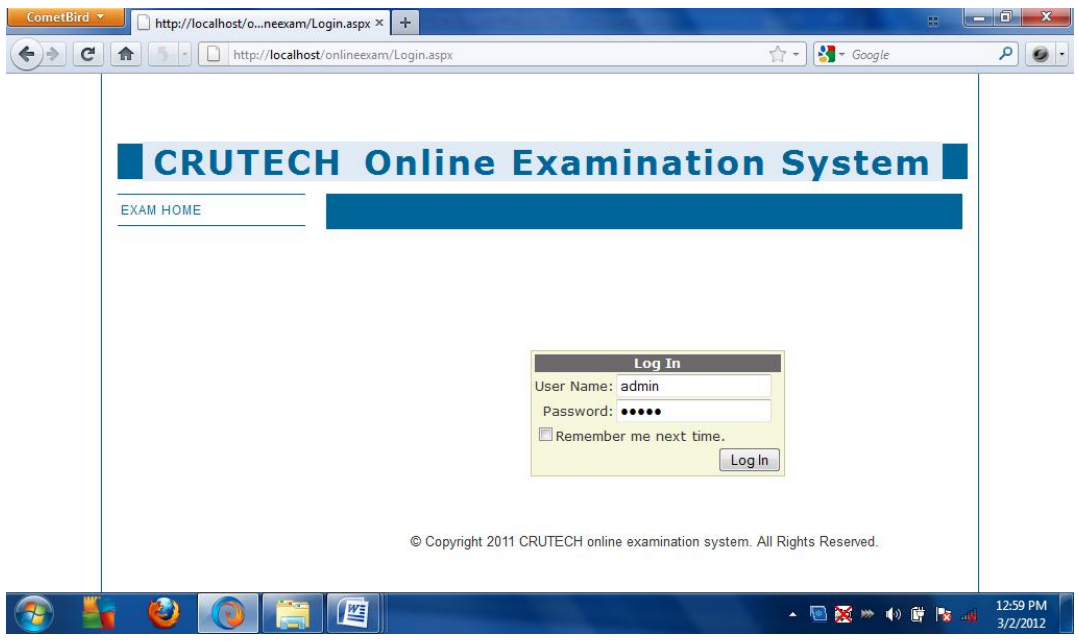

\section{User Login Screenshot}

This page allows student to enter exam pin has been generated for him the exam administrator. 


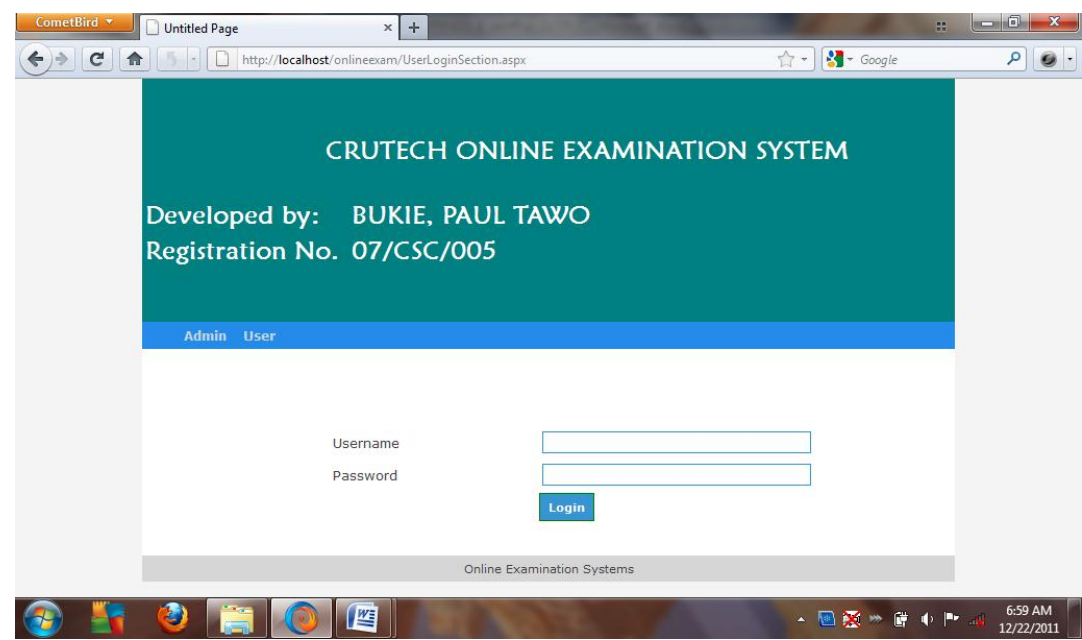

\section{Instruction Screenshot}

This page enable users to read examination instructions before starting exams.

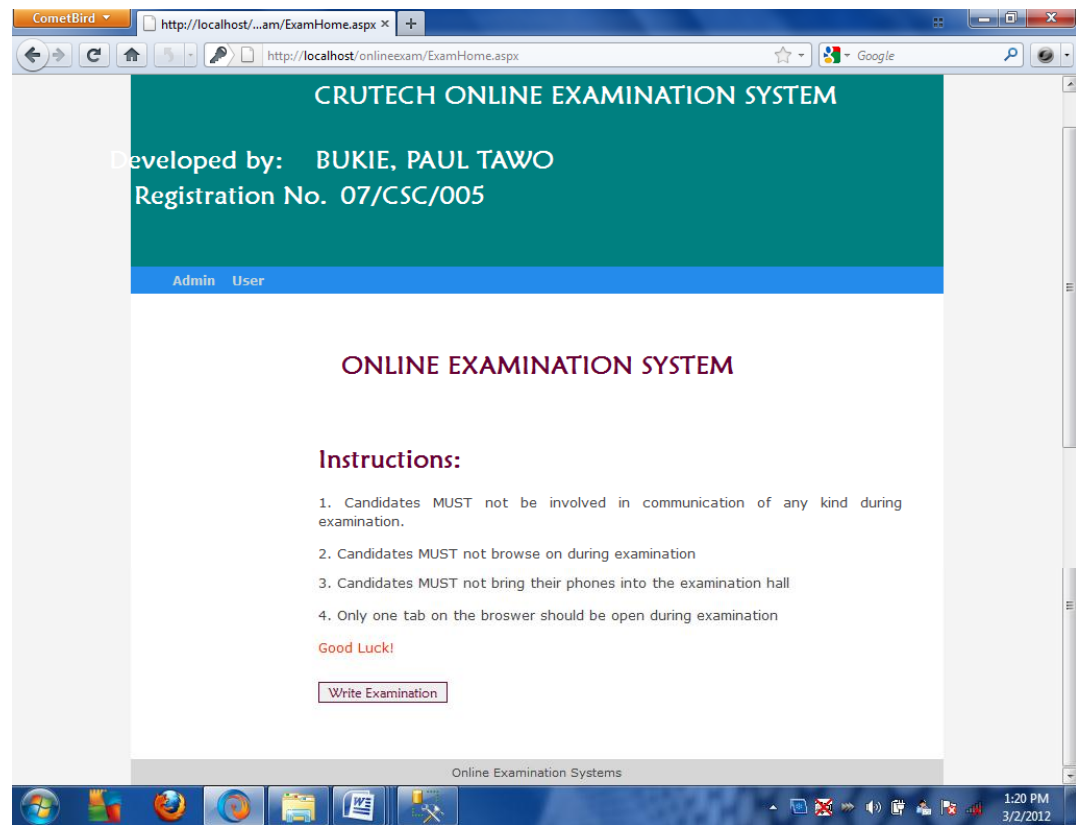

\section{Select Exam Screenshot}

This page allows the student to select exam to write. It has a dropdown menu where the student can choose a particular exam to take. The screenshot is shown below: 


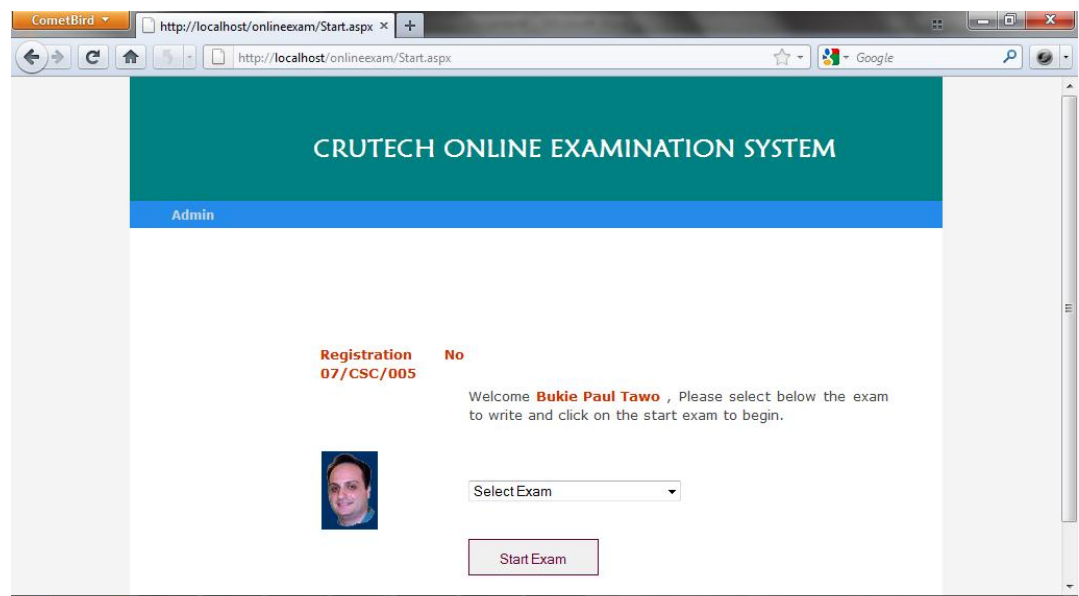

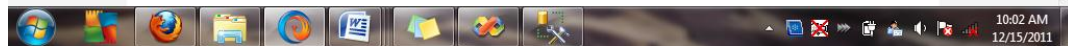

\section{Write Exam Screenshot}

This page is the main exam section. It serves exams questions to students. Below is the screen shot.

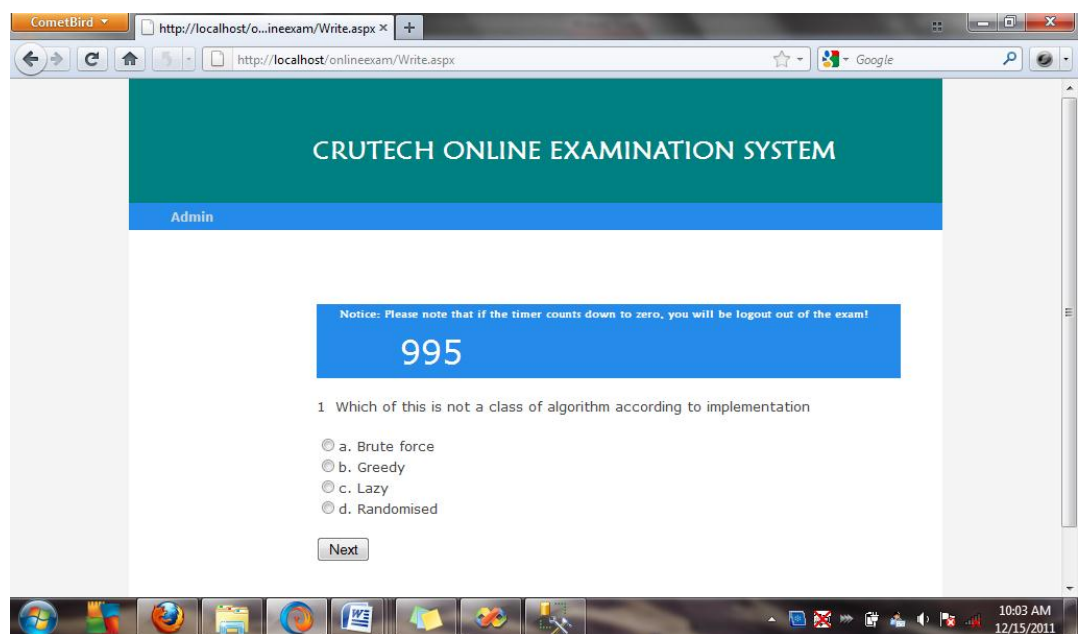

\subsubsection{Preliminary Result Screenshot}

This page shows the result of the examination after which the score is sent to the score table in the database. 


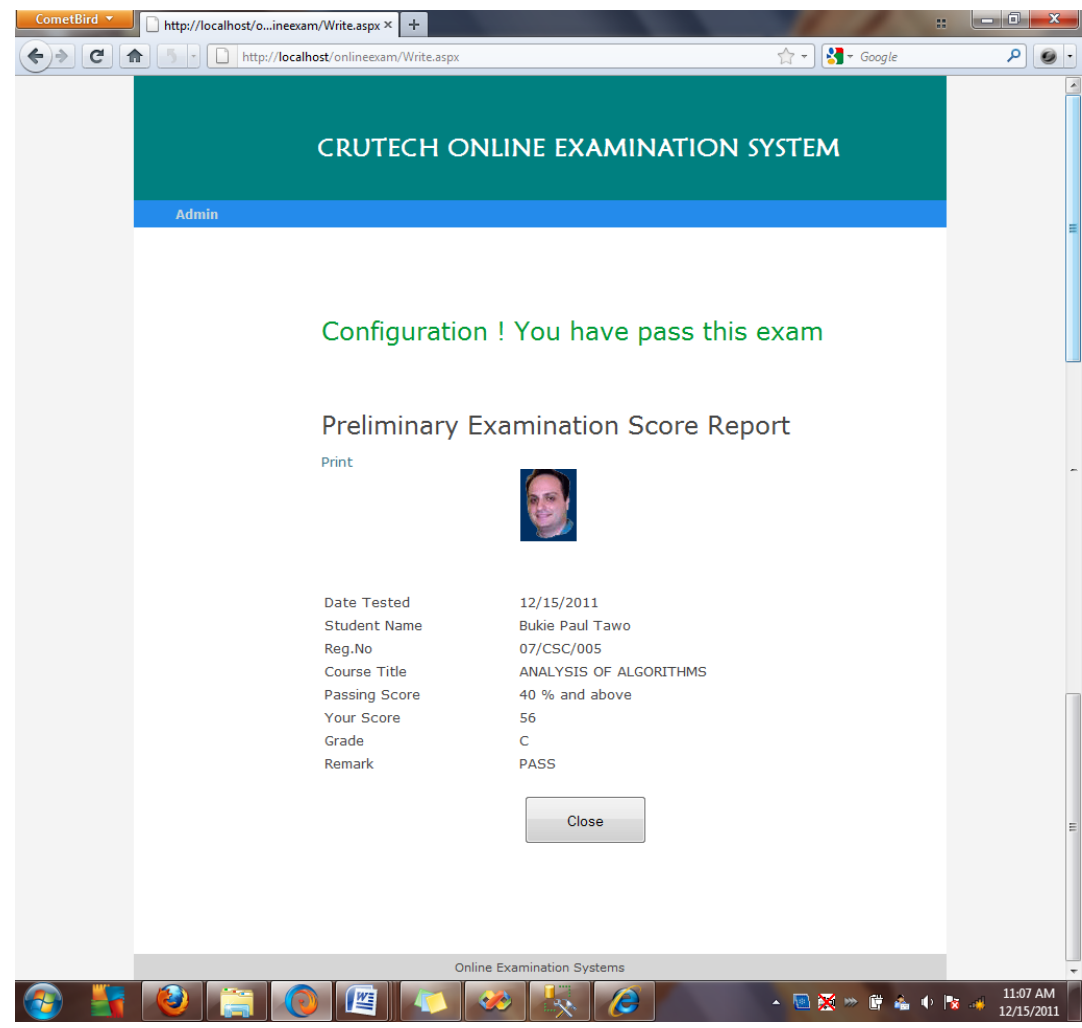

\section{View Result Screenshot}

This page enable administrator to view the scores of all the students that take the exams.

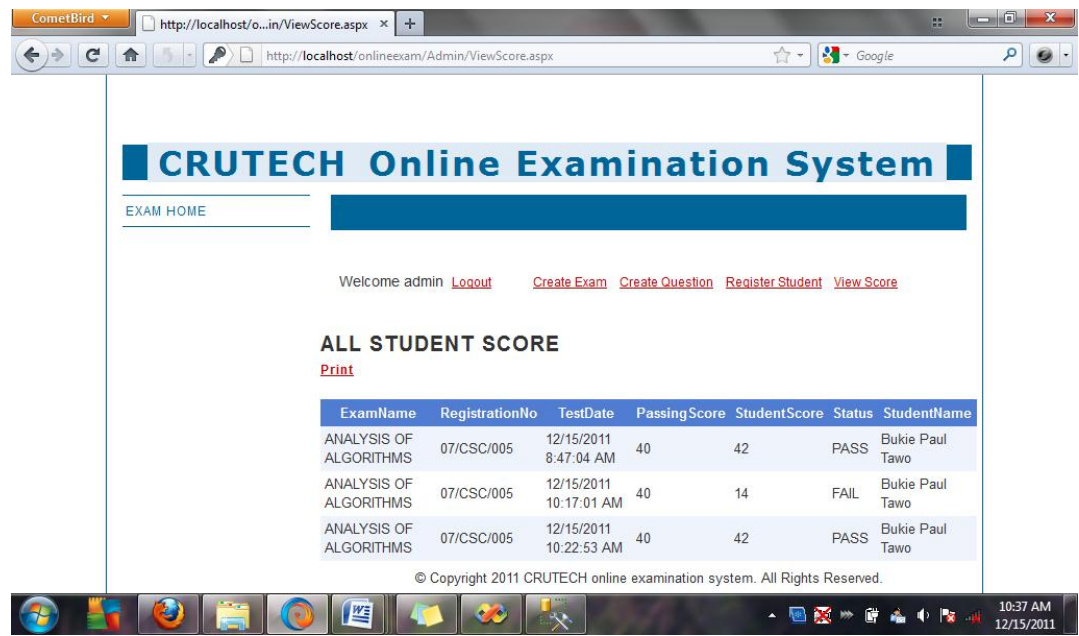

\section{CONCLUSION}

The Latin maxim "QUIDQUID INITIUM HABET FINEM HABET" means "WHATEVER HAS A BEGINNING HAS AN END". This research work is in its concluding stage. The importance of research work in academics cannot be overemphasized. It broadens the horizon of students in their area of studies with focus on the research topic.

In this research work, we have developed an overall solution to the examination administration problem in CRUTECH. It provides a user friendly platform of multiple choice questions examination. It can be used for academic purpose, professional certifications, staff promotion examination, Post UTME entrance examination, etc.

The online examination system is developed using Visual Studio 2008 with a linking technology called ASP.NET. We have used Microsoft SQL Server 2005 for the database design. The actual coding was done using $\mathrm{C \#}$. The system has reached a steady state where all 
bugs have been eliminated. The system would be operated at a high

level of efficiency and all the teachers and user associated with the system understands its advantage. The system solves the problem it was intended to solve as requirement specification.

\section{REFERENCES}

George Shepherd., 2005. Microsoft ASP.NET 2005: Step By Step. $5^{\text {th }}$ Ed. Ben Ryan and Kathleen Atkins (Eds.). Redmond Microsoft Press.
Jay Hilyard and Stephen Teilhet., 2008. C\# 3.0 Cookbook: More than 250 Solutions for C\# 3.0 Programming. ( $3^{\text {rd }}$ Ed.) United States of America: O'Reilly and Associates.

Jesse Liberty and Dan Hurwitz., 2002. Programming ASP.NET. Ron Petrusha and Valerie Quercia (Eds.) United States of America: O'Reilly and Associates

Stephen Walther., 2008. ASP.NET 3.5 Unleashed. United States of America: Sams Publishing

William B. Sanders., 2009. ASP.NET 3.5: A Beginner's Guide United States of America: McGraw-Hills. 